\title{
Association of serum levels of deoxyribose 1-phosphate and S-lactoylglutathione with neoadjuvant chemotherapy sensitivity in patients with gastric cancer: A metabolomics study
}

\author{
DAGUANG WANG ${ }^{1}$, WEI LI ${ }^{1}$, LEI YIN ${ }^{2}$, YECHAO DU $^{1}$, SHAOPENG ZHANG ${ }^{1}$ and JIAN SUO ${ }^{1,3}$ \\ ${ }^{1}$ Department of Gastrointestinal Surgery and ${ }^{2}$ Research Institute of Translational Medicine; \\ ${ }^{3}$ Jilin Province Key Laboratory of Bioinformatics for Gastrointestinal Tumor, \\ First Hospital of Jilin University, Changchun, Jilin 130021, P.R. China
}

Received March 23, 2019; Accepted January 7, 2020

DOI: $10.3892 / \mathrm{ol} .2020 .11350$

\begin{abstract}
The present study screened serum samples from patients with advanced-stage gastric cancer and known sensitivities to neoadjuvant chemotherapy, in order to identify metabolites that may serve as potential biomarkers for chemotherapy sensitivity. A total of 47 patients with stage III (T4b) or IV gastric cancer, including 31 in the training group and 16 in a validation group, were classified based on their responses to conversion therapy consisting of oxaliplatin, tegafur and continuous hyperthermic peritoneal perfusion with cisplatin. Serum samples were analyzed by liquid chromatography-mass spectrometry to obtain a metabolite profile of each patient. Patients who were responsive and non-responsive to neoadjuvant chemotherapy exhibited significant differences in serum levels of deoxyribose 1-phosphate, S-lactoylglutathione, lysophosphatidylcholine (16:0) and O-arachidonoyl ethanolamine. Logistic regression analysis indicated that deoxyribose 1-phosphate and S-lactoylglutathione were independently associated with chemosensitivity. Serum levels of deoxyribose 1-phosphate and S-lactoylglutathione were independently associated with the sensitivity of gastric cancer to neoadjuvant chemotherapy, therefore, serving as potential predictors of patient response.
\end{abstract}

\section{Introduction}

Gastric cancer is one of the most common malignancies worldwide, however this is highly variable in terms of geographical distribution (1). In 2012, global statistics for gastric cancer

Correspondence to: $\mathrm{Dr}$ Shaopeng Zhang or Dr Jian Suo, Department of Gastrointestinal Surgery, First Hospital of Jilin University, 71 Xinmin Street, Changchun, Jilin 130021, P.R. China E-mail: 958357004@qq.com

E-mail: suojian42@gmail.com

Key words: gastric cancer, neoadjuvant chemotherapy, metabolomics suggest that China currently accounts for $42 \%$ of all newly-diagnosed cases $(2,3)$. The 5-year survival rate in 2012 was $90 \%$ for patients with early-stage gastric cancer; however, the survival rate decreases to $<60 \%$ for patients with advanced-stage cancer (4). In particular, patients receiving palliative surgery for stage IV gastric cancer with peritoneal metastasis or for advanced-stage gastric cancer with local invasion have poor prognoses globally (5-7). Neoadjuvant chemotherapy is an effective approach for treating advanced-stage cancer, because it can lead to downgrading of the cancer stage and increase the median survival time of patients $(8-10)$. However, there are limited options for patients who present chemotherapy resistance; a relatively common phenomenon. In particular, the overall effective rate of neoadjuvant chemotherapy for gastric cancer is $\sim 45 \%$ (11), therefore identification of patients who may present neoadjuvant chemotherapy resistance remains a challenge.

A number of preclinical studies have identified altered metabolic pathways in tumors with various biological behaviors, and clinical studies have reported alterations in certain metabolites during the development and progression of colorectal, ovarian and endometrial cancer (12-16). For example, Furberg et al (12) reported that Low HDL-C, as part of the metabolic syndrome, is associated with increased postmenopausal breast cancer risk. Healy et al (13) found that metabolic syndrome and central obesity are common in patients with postmenopausal breast cancer, and that metabolic syndrome may be associated with a more aggressive tumor biology. Some of these alterations may serve as useful tumor response biomarkers. For example, the serum level of low-density lipoprotein-derived lipids predicts the response of patients with colorectal cancer to capecitabine (17). Patients with metastasis often present with metabolomic fingerprints that are associated with insensitivity or adverse effects to chemotherapies (18). A study of gastric cancer have demonstrated that certain lipid and carbohydrate metabolites, including 2,4-hexadienoic acid, 4-methylphenyl dodecanoate and glycerol tributanoate, are associated with pathological type, differentiation, location, staging and prognosis (19). However, to the best of our knowledge, the association of metabolite profiles with 
sensitivity of gastric cancer to neoadjuvant chemotherapy is unknown.

In the present study, sera from patients with gastric cancer who exhibited different sensitivities to chemotherapy were collected. Subsequently, serum samples were analyzed using liquid chromatography-mass spectrometry (LC-MS) to identify metabolites that were associated with sensitivity to neoadjuvant chemotherapy, and, therefore, have the potential to function as clinical markers for sensitivity to chemotherapy.

\section{Materials and methods}

Patient sample selection. The present retrospective study was approved by The Ethics Committee of the First Hospital of Jilin University (Changchun, China), and all patients provided written informed consent. All 47 patients (age range: 33-74 years old; 35 males and 12 females), including 31 in the training group and 16 in the validation group, were diagnosed with stage III (T4b) or IV gastric cancer and received conversion therapy, which was defined as R0 resection with unresected metastases, at the Department of Gastrointestinal Surgery at the First Hospital of Jilin University. Sample collection was conducted between August 2009 and October 2017. Diagnosis was based on pathological examination and abdominal imaging or laparoscopy. The inclusion criteria were as follows: i) $<75$ Years of age; ii) normal functions of the bone marrow, liver, heart and kidney; iii) primary gastric cancer with malignancy confirmed by pathological analysis; iv) signed informed consent form and agreement to receive neoadjuvant chemotherapy consisting of oxaliplatin, tegafur and continuous hyperthermic peritoneal perfusion (CHPP) of cisplatin; v) surgery following chemotherapy treatment; and vi) no other malignancies, immunosuppressive disorders or severe diseases affecting other organs. Patients were excluded if they had a congenital disorder, severe organic disease, gastrointestinal bleeding, perforation or infection, history of resection or palliative surgery, or received radiochemotherapy or biomedical therapy. All included patients were followed up until the end of chemotherapy and surgery.

Treatment, response evaluation and subgrouping. All patients received neoadjuvant chemotherapy, with each cycle consisting of intravenous oxaliplatin $\left(130 \mathrm{mg} / \mathrm{m}^{2}\right.$ on day 1$)$, CHPP of cisplatin $\left(50 \mathrm{mg} / \mathrm{m}^{2}\right.$ in 21 of $41^{\circ} \mathrm{C}$ saline at $35-45 \mathrm{ml} / \mathrm{min}$ on day 3 or 4) and orally-administered tegafur (40-60 mg twice/day; days 1-14), followed by a 7-day break. Patients received 3-5 cycles, and responses were evaluated every two cycles using enhanced abdominal $\mathrm{CT}$ according to the criteria of Response Evaluation Criteria in Solid Tumors (20). Therefore, complete response (CR) indicated complete disappearance of the tumor; partial response (PR) indicated $\mathrm{a} \geq 30 \%$ reduction of the tumor; progressive disease (PD) indicated a $\geq 20 \%$ increase of the tumor or the appearance of new lesions; and stable disease (SD) indicated the state between PR and PD. Patients with CR and PR were summed to calculate the overall response rate, and were defined as the chemo-sensitive group; those with SD and PD were classified as the chemoresistant group. Pathological analysis was performed following surgery and results were classified according to the criteria of the Japanese Association of Medical Sciences. Therefore, grade 0 indicated no evidence of treatment response; grade 1a indicated tumor cells is visible in $>2 / 3$ examination area; grade $1 b$ indicated tumor cells is visible in $1 / 3-2 / 3$ examination area; grade 2 indicated tumor cells is visible in $<1 / 3$ examined area; grade 3 indicated no residual tumor cells. The adverse effects of chemotherapy were also assessed using the Common Terminology Criteria for Adverse Events (21).

Sample collection and LC-MS. Prior to chemotherapy, a fasting blood sample was taken from the cubital vein of each patient on day 1 of chemotherapy. The sample was centrifuged at 3,500 x g/min for $5 \mathrm{~min}$ at room temperature, and the supernatant was collected and stored at $-80^{\circ} \mathrm{C}$. The frozen serum sample was allowed to thaw for $20 \mathrm{~min}$ at room temperature prior to analysis. As a quality control (QC), $20 \mu \mathrm{l}$ of each of the 31 samples in the training group, consisting of 16 chemo-sensitive and 15 chemoresistant patients, were pooled and vortexed. Subsequently, acetonitrile (500 $\mu \mathrm{l})$ was added to $100 \mu \mathrm{l}$ of each of the 31 samples and to the QC, followed by mixing, centrifugation at $14,000 \mathrm{x} \mathrm{g} / \mathrm{min}$ for $5 \mathrm{~min}$ at $4{ }^{\circ} \mathrm{C}$, and collection of the supernatants for LC-MS analysis. Identical techniques were used for samples in the training and validation groups. LC-MS analysis was performed using the AB Sciex TripleTOF 5600 system (Sciex), according to the manufacturer's protocols. The column was an Eclipse Plus C18 (2.1x150 mm; pore size, $3.5 \mu \mathrm{m}$; Agilent Technologies, Inc.) and was maintained at $45^{\circ} \mathrm{C}$ during separation. The mobile phases in positive ion mode were $0.1 \%$ formic acid in (A) water and (B) acetonitrile; the mobile phases in negative ion mode were (A) water and (B) acetonitrile. Samples were eluted with $80 \%$ A and $20 \%$ B for the first 3 min, a gradient from $20 \%$ B to $95 \%$ B over 6 min, followed by $95 \%$ B for $1.5 \mathrm{~min}$. Then the percentage of B was dropped to $20 \%$ within $0.1 \mathrm{~min}$ and maintained for $1.4 \mathrm{~min}$. The flow rate was constant at $900 \mu \mathrm{l} / \mathrm{min}$. Mass spectrometry was performed using the AB Sciex TripleTOF 5600 system, which was fitted with an electrospray ionization source operating in positive and negative ion modes. Nitrogen was used as a nebulizer and cone gas The MS acquisition used TOF MS-IDA-MS/MS mode. The scan period contains TOF MS scan and product ion scan based on information dependent acquisition (IDA). TOF MS range was 50-1,000 m/z, MS/MS (product ion) range-(5 MS/MS) was $50-1,000 \mathrm{~m} / \mathrm{z}$; The other MS parameters were as follows: Nebulizer (50 psi), heater and curtain gas flow rates 50,50 and 30 units, respectively; ionspray needle voltage $4500 \mathrm{~V}$; heater gas temperature $450^{\circ} \mathrm{C}$. Declustering potential $(\mathrm{V})$ and collision energy $(\mathrm{eV})$ were: 100, 10 for the TOF MS scan experiment; Declustering potential (V), collision energy (eV) and CES were 100,30, 15 for the MS/MS scan experiment. The instrument was calibrated prior to analysis according to the manufacturer's protocols.

Statistical analysis. The LC-MS data were acquired using Analyst 1.5.1 software (AB Sciex LLC) and processed using PeakView software version 1.1 (AB Sciex LLC). Normalization, scaling, noise filtering and peak alignment were performed using MarkerView software version 1.2.1 (AB Sciex LLC) prior to principal component analysis (PCA) and comparisons using an unpaired $\mathrm{t}$-test. $\mathrm{P}<0.05$ was considered to indicate a statistically significant difference. Data are presented as mean \pm standard deviation. To identify metabolites that differed 
Table I. Clinicopathological characteristics of the patients.

\begin{tabular}{|c|c|c|c|c|c|c|}
\hline \multirow[b]{2}{*}{ Variables } & \multicolumn{3}{|c|}{ Training group } & \multicolumn{3}{|c|}{ Validation group } \\
\hline & Sensitive, $\mathrm{n}$ & Resistant, $\mathrm{n}$ & $\mathrm{P}$-value & Sensitive, $\mathrm{n}$ & Resistant, $\mathrm{n}$ & P-value \\
\hline Patients & 16 & 15 & & 8 & 8 & \\
\hline Age, years & $58.06 \pm 2.39$ & $55.53 \pm 3.19$ & 0.237 & $62.38 \pm 1.97$ & $54.13 \pm 5.66$ & 0.190 \\
\hline \multicolumn{7}{|l|}{ Sex, $n$} \\
\hline Male & 11 & 12 & 0.685 & 6 & 6 & 0.715 \\
\hline Female & 5 & 3 & & 2 & 2 & \\
\hline \multicolumn{7}{|l|}{ T classification ${ }^{\mathrm{a}}$} \\
\hline $\mathrm{T} 1$ & 0 & 0 & 0.323 & 0 & 0 & 0.282 \\
\hline $\mathrm{T} 2$ & 1 & 0 & & 0 & 0 & \\
\hline $\mathrm{T} 3$ & 7 & 4 & & 1 & 4 & \\
\hline $\mathrm{T} 4$ & 8 & 11 & & 7 & 4 & \\
\hline \multicolumn{7}{|c|}{$\mathrm{N}$ classification ${ }^{\mathrm{a}}$} \\
\hline No & 1 & 0 & 0.670 & 0 & 0 & 0.435 \\
\hline N1 & 1 & 1 & & 3 & 3 & \\
\hline $\mathrm{N} 2$ & 9 & 7 & & 4 & 2 & \\
\hline N3 & 5 & 7 & & 1 & 3 & \\
\hline \multicolumn{7}{|c|}{ M classification ${ }^{\mathrm{a}}$} \\
\hline M0 & 4 & 5 & 0.704 & 2 & 2 & 0.715 \\
\hline M1 & 12 & 10 & & 6 & 6 & \\
\hline \multicolumn{7}{|l|}{ Differentiation } \\
\hline Low & 10 & 12 & 0.433 & 5 & 3 & 0.310 \\
\hline Moderate & 6 & 3 & & 3 & 5 & \\
\hline \multicolumn{7}{|l|}{ Tumor size } \\
\hline$\geq 5 \mathrm{~cm}$ & 13 & 12 & 0.683 & 6 & 4 & 0.304 \\
\hline$<5 \mathrm{~cm}$ & 3 & 3 & & 2 & 4 & \\
\hline \multicolumn{7}{|l|}{ Tumor location } \\
\hline Antrum & 8 & 6 & 0.577 & 6 & 4 & 0.304 \\
\hline Body/fundus & 8 & 9 & & 2 & 4 & \\
\hline
\end{tabular}

${ }^{\mathrm{a}}(46)$. T, tumor; N, node; M, metastasis.

in patients with chemosensitivity and chemoresistance, an integrated software system (BRB-Array Tools version 3.3.0; linus.nci.nih.gov/BRB-ArrayTools.html) was used to conduct hierarchical clustering analysis of samples from the 31 patients in the training group. The metabolites were identified using the Human Metabolome Database (hmdb.ca). SPSS 20.0 software (IBM Corp.) was used to generate receiver operating characteristic (ROC) curves. AUC values above the cut-off value of 0.8 (higher concentrations in chemo-sensitive patients) or below the cut-off value of 0.2 (higher concentrations in chemo-resistant patients) were selected. Following analysis of the samples in the training group, samples of the 16 patients in the validation group were used for differentiation and clustering analysis. Tree clusters and shorter Euclidean distances indicate the greater similarities between samples or metabolites. SPSS was used to perform logistic regression analysis to determine the associations of cancer chemosensitivity with age, sex, tumor size, tumor location, tumor stage, tumor differentiation, vascular invasion and the identified metabolites.

\section{Results}

Response to chemotherapy and adverse effects. The clinicopathological characteristics of patients in the training group and validation group were initially compared, and patients were divided into the chemo-sensitive and chemo-resistant groups (Table I). There were no significant differences identified in age, sex, tumor location, tumor size, or tumor-node-metastasis classification. Pathological classification, which was performed according to the criteria of the Japanese Association of Medical Sciences (22-25), indicated that there were 3 patients with grade 1a, 20 patients with grade $1 \mathrm{~b}, 19$ patients with grade 2 , and 5 patients with grade 3 disease (data not shown).

The adverse effects of chemotherapy were also assessed using the Common Terminology Criteria for Adverse Events (21). The results revealed that 10 patients $(21.3 \%)$ had grade I leukopenia, 4 patients $(8.5 \%)$ had grade II leukopenia and 2 patients $(4.3 \%)$ each had grade I and II thrombocytopenia. 
Table II. Metabolites identified that differentiate between chemo-sensitive and chemo-resistant gastric cancer.

\begin{tabular}{lcccccc}
\hline & & & \multicolumn{2}{c}{ Signal intensity } & & \\
\cline { 4 - 5 } Metabolite & $\mathrm{m} / \mathrm{z}$ & Retention & Sensitive & Resistant & Weight (\%) & P-value \\
\hline Deoxyribose 1-phosphate & 215.0315 & 9.52 & $7,598.48 \pm 955.87$ & $2,482.61 \pm 811.16$ & 100 & $0.00141^{\mathrm{b}}$ \\
S-Lactoylglutathione & 380.1122 & 11.3 & $312.26 \pm 148.42$ & $855.95 \pm 103.00$ & 100 & $0.00616^{\mathrm{b}}$ \\
PC [15:0/16:1(9Z)] & 718.5128 & 8.46 & $419.98 \pm 152.03$ & $817.05 \pm 101.27$ & 100 & $0.04175^{\mathrm{a}}$ \\
LysoPC (16:0) & 496.3331 & 8.78 & $6,637.71 \pm 2345.54$ & $1,033.40 \pm \pm 342.01$ & 100 & $0.0287^{\mathrm{a}}$ \\
O-Arachidonoyl Ethanolamine & 431.3109 & 9.48 & $694.53 \pm 94.61$ & $316.66 \pm 39.18$ & 100 & $0.00180^{\mathrm{b}}$ \\
Unknown metabolite & 654.4363 & 7.05 & $427.61 \pm 115.87$ & $49.90 \pm 20.11$ & 80 & $0.00475^{\mathrm{b}}$ \\
3-(3,5-Diiodo-4- & 434.8173 & 8.13 & $3.28 \pm 2.28$ & $75.10 \pm 34.81$ & 80 & $0.04223^{\mathrm{a}}$ \\
hydroxyphenyl) lactate & & & & & &
\end{tabular}

${ }^{\mathrm{a}} \mathrm{P}<0.05,{ }^{\mathrm{b}} \mathrm{P}<0.01$. $\mathrm{PC}$, phosphatidylcholine.

In addition, 8 patients (17\%) had grade I nausea or vomiting, and 2 patients $(4.3 \%)$ had grade II nausea or vomiting in 47 patients from the training and validation groups. No patients had severe complications or required hospitalization.

Metabolomic profiles of chemo-sensitive and chemo-resistant patients. Representative total ion current chromatograms of metabolites in the sera of chemo-sensitive patients (Fig. 1A and C) and chemo-resistant patients (Fig. 1B and D) were analyzed. These results indicated differences in the levels of multiple metabolites in the positive ion mode (Fig. 1A and B) and negative ion mode (Fig. 1C and D). The difference does not include the P-value, which provides a reference for the subsequent data analysis. Additionally, the metabolomic profiles of 31 patients in the training group were compared using PCA (Fig. 2). The results demonstrated a clear separation of the sera of chemo-sensitive patients, chemo-resistant patients and the QC sample in the positive ion mode (Fig. 2A) and negative ion mode (Fig. 2B). The difference in type and number of metabolites determined the dispersion of the samples. The difference in metabolites in the serum samples was larger, with a greater distance between samples. By contrast, the smaller the difference in metabolites in serum samples, the closer the distance between samples. The QC sample consisted of $20 \mu 1$ of each of the 31 samples in the training group. The metabolites in the samples of the QC group were not significantly different and the aggregation degree of these samples reflected the reliability of the experimental results. It was indicated that the distance between samples within each group was small and therefore the difference in metabolites in all three groups was small (Fig. 2). However, the distance of the samples between groups was large, indicating that the difference in metabolites between groups was large. To evaluate the experimental accuracy of the present study, at least one sample from the QC group for every six samples was assessed. Since the serum samples were limited, in experiment B, six QC samples completely met the requirements of the experiment. Experiment A utilized the remaining nine samples of the QC group. The present study subsequently normalized, scaled and noise-filtered the raw data, and aligned the chromatograms using MarkerView.
Comparison of the chemo-sensitive and chemo-resistant groups indicated significant differences for 255 metabolites in the positive ion mode (Table SI) and 64 metabolites in the negative ion mode (Table SII) (both $\mathrm{P}<0.05$ ).

Metabolites that differentiate between chemo-sensitive and chemo-resistant patients. Further screening was performed using support vector machines of BRB-array Tools for the 31 patients in the training group to identify differentiating metabolites with weights of $\geq 80 \%$ (Table II). Based on the Human Metabolome Database the following seven metabolites met the criteria of the present study: Deoxyribose 1-phosphate, S-lactoylglutathione, phosphatidylcholine (PC) [15:0/16:1(9Z)], lysoPC (16:0), O-arachidonoyl ethanolamine, 3-(3,5-diiodo-4-hydroxyphenyl) lactate and an unknown metabolite (Table II). We found no metabolites associated with 654.4363 . Maybe 654.4363 is a new metabolites not included in the database. Based on the measured levels of these seven metabolites, 14/16 chemo-sensitive patients were classified as sensitive, and 13/15 chemo-resistant cases as resistant (Table III). Therefore, this procedure had a sensitivity of $87.5 \%$ and a specificity of $86.7 \%$.

Hierarchical clustering analysis was also performed for these seven metabolites to assess the similarity of the metabolomic profiles of patients with similar sensitivities to chemotherapy (Fig. 3). The results indicated that the concentrations of these seven metabolites separated the 31 patients in the training group into predominantly chemo-sensitive and chemo-resistant subgroups. ROC analysis and calculated areas under the curves (AUCs) were utilized to evaluate the performance of each metabolite as a marker for cancer chemosensitivity (Fig. 4A; Table IV). The results indicated that four metabolites, including deoxyribose 1-phosphate, S-lactoylglutathione, lysoPC (16:0) and $\mathrm{O}$-arachidonoyl ethanolamine, had AUC values above the cut-off value of 0.8 (higher concentrations in chemo-sensitive patients) or below the cut-off value of 0.2 (higher concentrations in chemo-resistant patients; Table IV). Analysis of the average concentrations of these four metabolites indicated significant differences in patients with chemo-sensitive and chemo-resistant tumors (Fig. 4B). Fig. S1 shows random images and levels of four metabolites in the chemo-resistant and chemo-sensitive groups. 

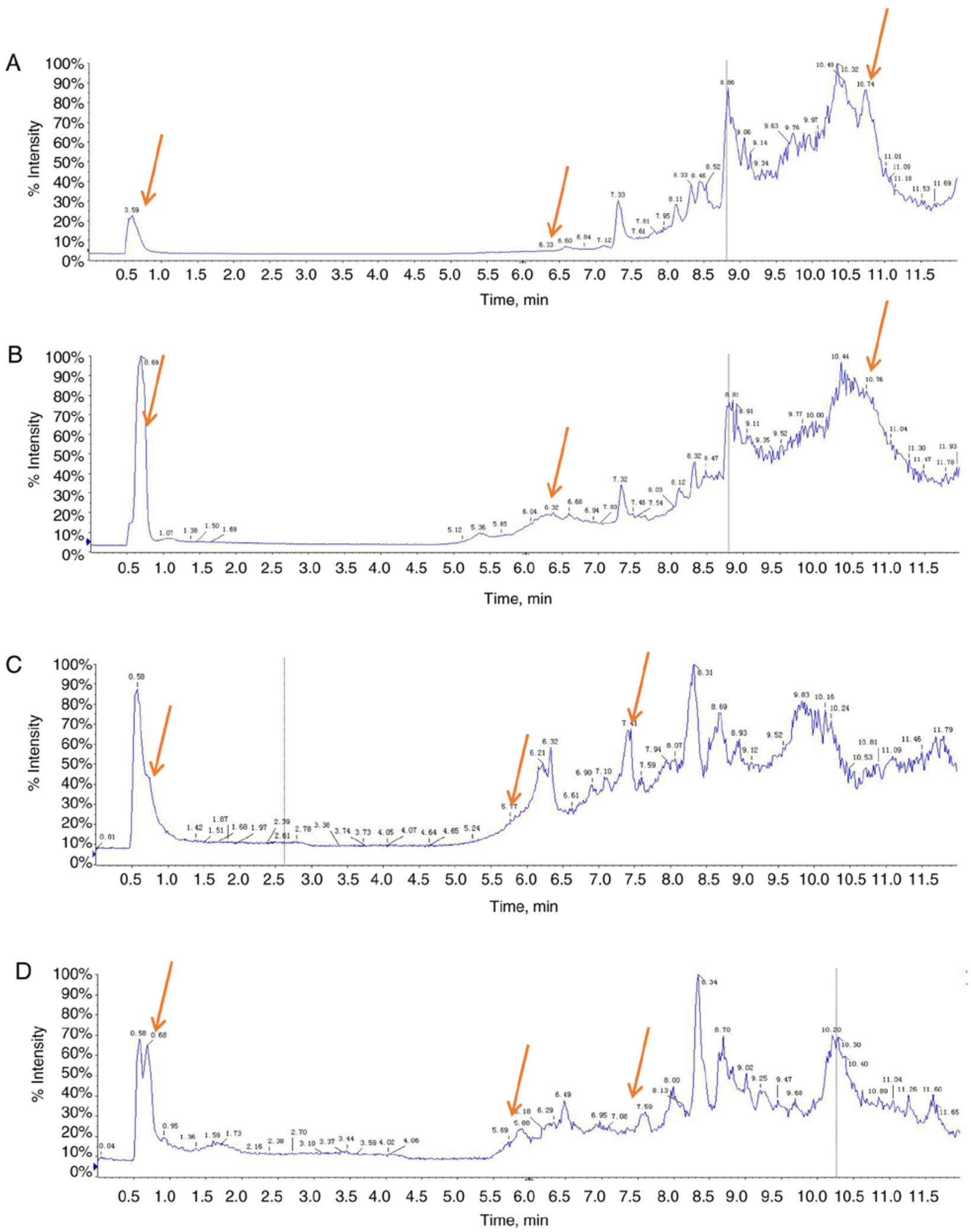

Figure 1. TIC chromatograms of metabolites in the sera of four representative patients with gastric cancer in the training group. (A) TIC profiles of chemo-sensitive in the positive ion mode; (B) TIC profiles of chemo-resistant in the positive ion mode. (C) TIC profiles of chemo-sensitive in the negative ion mode; (D) TIC profiles of chemo-resistant in the negative ion mode. Red arrows indicate metabolites subsequently identified as having different abundances in the two groups. TIC, total ion current.

Analysis of the validation group. Hierarchical clustering analysis was subsequently used to analyze the metabolome data of the 16 patients in the validation group using the same four metabolites identified in the ROC analysis for the training group (Fig. 5). This analysis separated these 16 patients into two subgroups: One subgroup included 7 chemo-sensitive patients and 1 chemo-resistant patient and the other subgroup included 7 chemo-resistant patients and 1 chemo-sensitive patient. This 


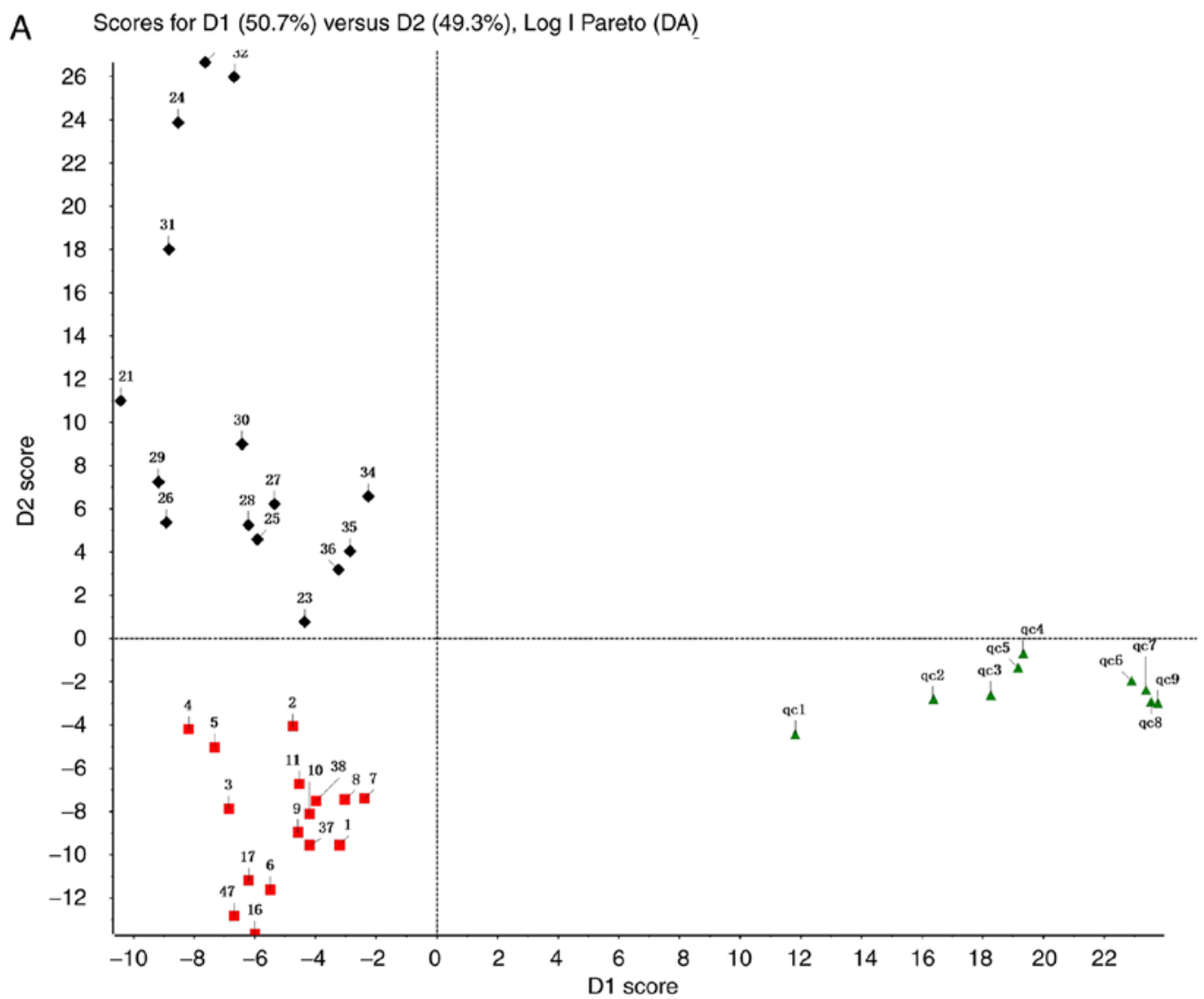

B

Scores for D1 (50\%) versus D2 (50\%), Log I Pareto (DA)

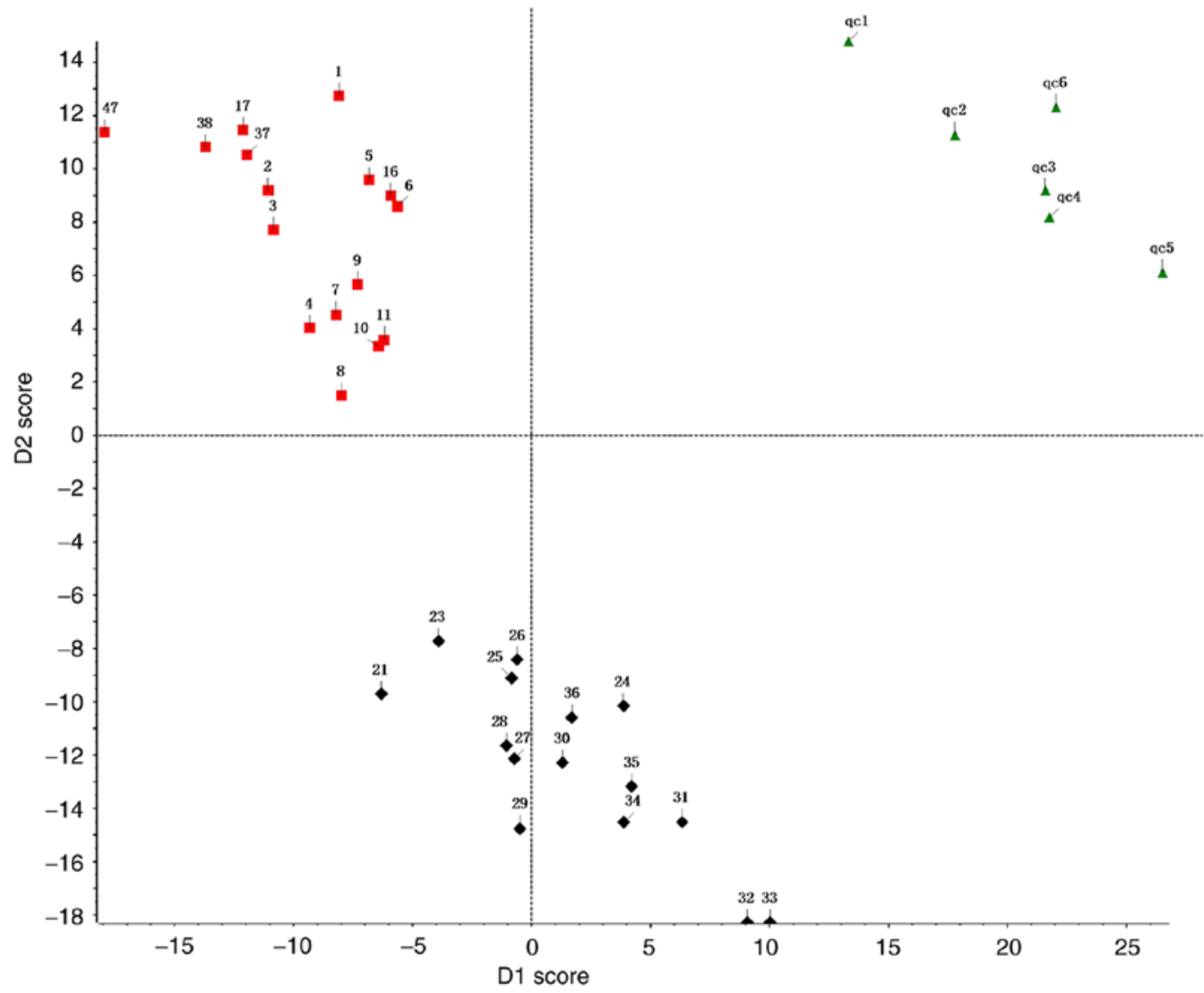

Figure 2. Principal component analysis of serum samples from the training group ( $\mathrm{n}=31)$ and the $\mathrm{QC}$ sample using the (A) positive ion mode and (B) negative ion mode. Log I Pareto (DA) is a PCA method, which refers to the analysis in Pareto mode after Log conversion. Each dot represents a single sample. Red square represents the chemo-sensitive group, the black diamond represents the chemo-resistant group, and the green triangle represents the QC group. QC, quality control. 
Table III. Performance of seven identified metabolites [deoxyribose 1-phosphate, S-lactoylglutathione, PC (15:0/16:1(9Z)), lysoPC (16:0), O-arachidonoyl ethanolamine, 3-(3,5-diiodo-4-hydroxyphenyl] lactate and an unknown metabolite), in differentiating between chemo-sensitive and chemo-resistant patients in the training group.

\begin{tabular}{|c|c|c|c|c|c|}
\hline \multirow[b]{2}{*}{ Prediction } & \multicolumn{2}{|c|}{ Response to chemotherapy } & \multirow[b]{2}{*}{ Total, $\mathrm{n}$} & \multirow[b]{2}{*}{ Sensitivity } & \multirow[b]{2}{*}{ Specificity } \\
\hline & Sensitive, $\mathrm{n}$ & Resistant, $\mathrm{n}$ & & & \\
\hline Sensitive & 14 & 2 & 16 & & \\
\hline Resistant & 2 & 13 & 15 & $87.5 \%$ & $86.7 \%$ \\
\hline Total & 16 & 15 & 31 & & \\
\hline
\end{tabular}

PC, phosphatidylcholine.

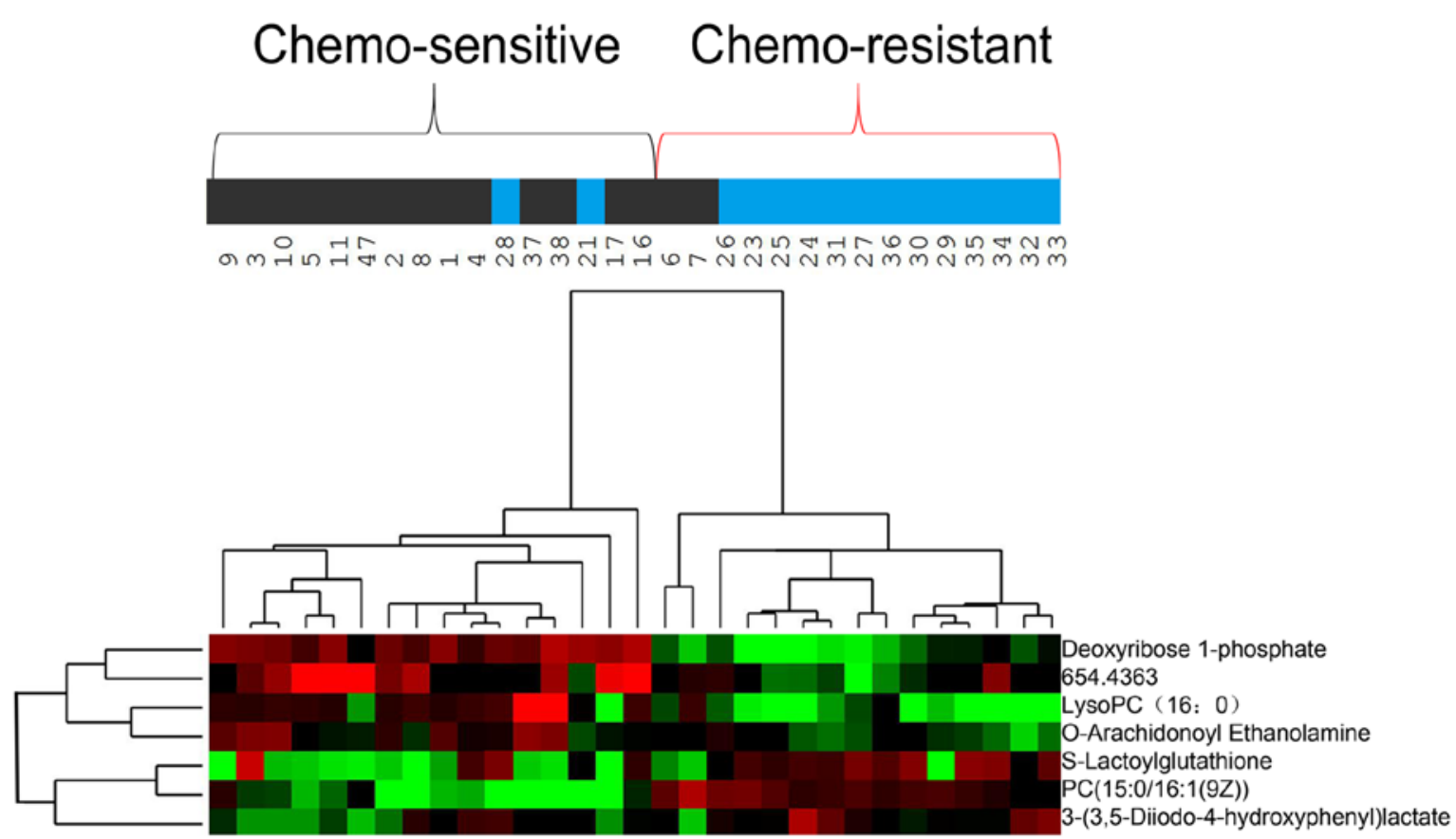

Figure 3. Hierarchical clustering analysis of metabolome data for seven metabolites in the training group $(\mathrm{n}=31)$. Metabolite levels were used to color code individual samples from green (low concentration) to red (high concentration), with grey denoting absence of the substance. Tree clusters and shorter Euclidean distances indicate greater similarities between samples or metabolites. The numbers represent the patient IDs. The blue color of the top bars represents the chemo-resistant group, and the black color of the top bars represents the chemo-sensitive group. PC, phosphatidylcholine.

classification of chemo-sensitive and chemo-resistant patients had a sensitivity and specificity of $82.5 \%$ (Table V).

Metabolites independently associated with cancer chemosensitivity. Logistic regression analysis was subsequently performed to assess the association of these four metabolites with multiple demographic and clinicopathological characteristics in all 47 patients. Univariate analysis demonstrated that out of the 4 metabolites, only deoxyribose 1-phosphate, S-lactoylglutathione, lysoPC (16:0) and O-arachidonoyl ethanolamine were associated with cancer chemosensitivity (Table VI). Multivariate logistic regression analysis, which was adjusted for confounding factors, including deoxyribose 1-phosphate, S-lactoylglutathione, lysoPC (16:0) and $\mathrm{O}$-arachidonoyl ethanolamine, indicated that deoxyribose 1-phosphate $(\mathrm{P}=0.007)$ and $\mathrm{S}$-lactoylglutathione $(\mathrm{P}=0.025)$ were independently associated with cancer chemosensitivity
(Table VII). The two other metabolites were lysoPC (16:0) and $\mathrm{O}$-arachidonoyl ethanolamine, but these are not shown in Table VII because they were not independently associated with cancer chemosensitivity.

\section{Discussion}

Although the incidence of gastric cancer has declined during the past decade, treatment remains challenging $(3,26,27)$. There have been recent improvements in the surgical and chemotherapeutic strategies for this malignancy (7,28-30). Surgical resection is the optimal approach, however it provides only limited help for patients with locally advanced disease, such as stage IV cancer with peritoneal metastasis (31). Neoadjuvant chemotherapy is an alternative approach. The results of two clinical trials MAGIC (8) and FNLCLCC/FFCD (15) suggested that perioperative chemotherapy significantly 
A
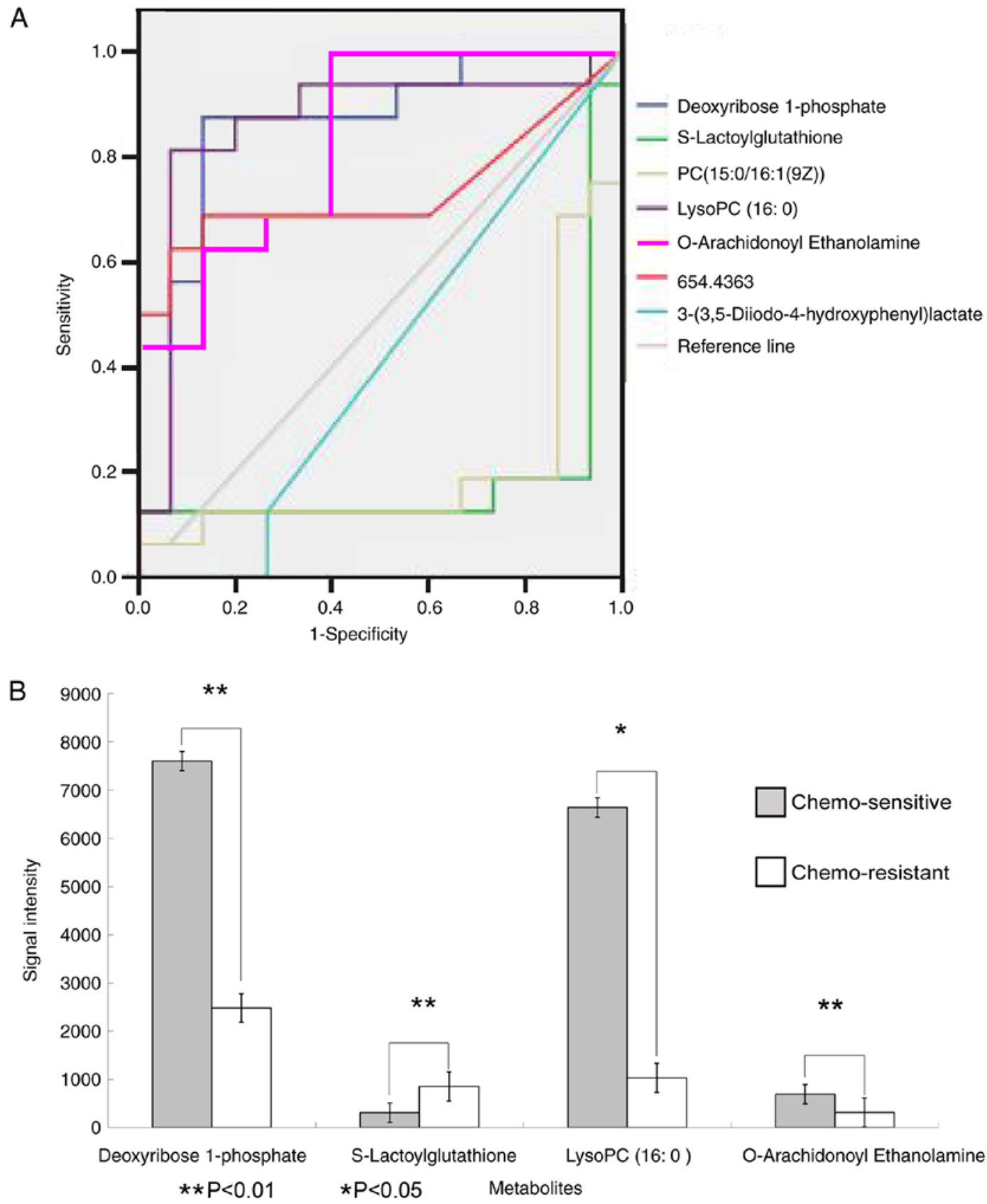

Figure 4. ROC curves of the seven metabolites and Levels of the four metabolites. (A) ROC curves of the diagnostic performance of each of the seven metabolites in the training group $(n=31)$ in predicting chemosensitivity. Curves closer to the top left corner are metabolites with higher concentrations in chemo-sensitive patients; curves closer to the bottom right corner are metabolites with higher concentrations in chemo-resistant patients. (B) Levels of the four metabolites identified by ROC analysis in patients with chemo-sensitive and chemo-resistant cancer. ROC, receiver operating characteristic; PC, phosphatidylcholine.

improves the overall survival time and progression-free survival rate of patients with gastric adenocarcinoma and esophagogastric junction adenocarcinoma. A previous study reported that neoadjuvant chemotherapy leads to downgrading of tumor classification and increased R0 resection rate (32). However, the overall efficiency of neoadjuvant chemotherapy is $<50 \%$ (11). Therefore, it is necessary to identify patients with favorable responses to chemotherapy. The results of the present study indicated that metabolomic analysis of the serum samples of patients with gastric cancer may serve as a potential predictor of patient sensitivity to neoadjuvant chemotherapy.

Identification of cancer biomarkers has been a major research focus in recent decades, and biomarkers, such as human epidermal growth factor receptor 2 in breast and gastric cancer, KRAS in colon and lung cancer, and epidermal growth factor receptor in colon and lung cancer, are now commonly used in clinical diagnosis (33-35). Due to the fact that chemotherapy response is associated with genetic and epigenetic alterations relevant to the metabolism of chemotherapeutic agents, endogenous metabolites also have the potential to function as biomarkers that can be easily and non-invasively analyzed (36). However, few studies have examined the use of small-molecule metabolites as diagnostic markers. The present study identified four serum metabolites as potential markers for sensitivity of gastric cancer to neoadjuvant chemotherapy: Deoxyribose 1-phosphate, 
Table IV. Summary of the results of ROC analysis for seven metabolites [deoxyribose 1-phosphate, S-lactoylglutathione, PC (15:0/16:1(9Z)), lysoPC (16:0), O-arachidonoyl ethanolamine, 3-(3,5-diiodo-4-hydroxyphenyl) lactate, and an unknown metabolite].

\begin{tabular}{lccccc}
\hline & & & & \multicolumn{2}{c}{$95 \%$ CI } \\
\cline { 4 - 6 } Variable & Area under the curve & Standard error & Stepwise significance & Lower & Upper \\
\hline Deoxyribose 1-phosphate & 0.854 & 0.074 & 0.001 & 0.709 & 0.999 \\
S-Lactoylglutathione & 0.163 & 0.084 & 0.001 & 0.001 & 0.328 \\
PC [15:0/16:1(9Z)] & 0.208 & 0.090 & 0.006 & 0.032 & 0.384 \\
LysoPC (16:0) & 0.863 & 0.077 & 0.001 & 0.693 & 1.000 \\
O-Arachidonoyl Ethanolamine & 0.833 & 0.072 & 0.002 & 0.692 & 0.975 \\
Unknown metabolite & 0.733 & 0.098 & 0.027 & 0.542 & 0.925 \\
3-(3,5-Diiodo-4-hydroxyphenyl)lactate & 0.413 & 0.105 & 0.406 & 0.207 & 0.618 \\
\hline
\end{tabular}

PC, phosphatidylcholine.

Table V. Performance of four identified metabolites, including, deoxyribose 1-phosphate, S-lactoylglutathione, lysoPC (16:0), and O-arachidonoyl ethanolamine, in differentiating between chemo-sensitive and chemo-resistant patients in the validation group.

\begin{tabular}{|c|c|c|c|c|c|}
\hline \multirow[b]{2}{*}{ Prediction } & \multicolumn{2}{|c|}{ Response to chemotherapy } & \multirow[b]{2}{*}{ Total, $\mathrm{n}$} & \multirow[b]{2}{*}{ Sensitivity } & \multirow[b]{2}{*}{ Specificity } \\
\hline & Sensitive, $\mathrm{n}$ & Resistant, $\mathrm{n}$ & & & \\
\hline Sensitive & 7 & 1 & 8 & & \\
\hline Resistant & 1 & 7 & 8 & $82.5 \%$ & $82.5 \%$ \\
\hline Total & 8 & 8 & 16 & & \\
\hline
\end{tabular}

PC, phosphatidylcholine.

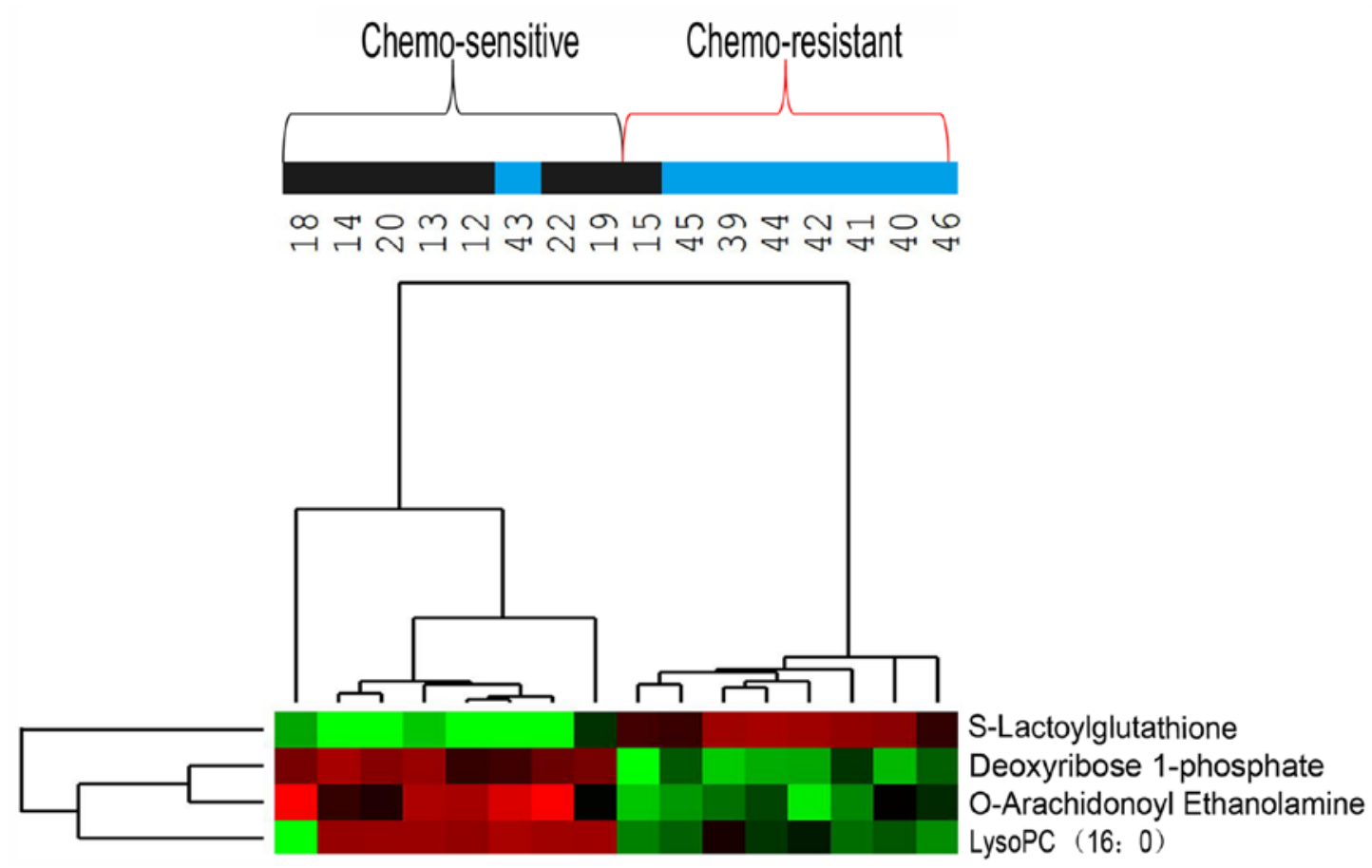

Figure 5. Hierarchical clustering analysis of metabolome data for four metabolites in the validation group ( $\mathrm{n}=16)$. Metabolite levels were used to color code individual samples from green (low concentration) to red (high concentration), with grey denoting absence of the substance. Tree clusters and shorter Euclidean distances indicate the greater similarities between samples or metabolites. The numbers represent the patient IDs. The blue color of the top bars represents the chemo-resistant group, and the black color of the top bars represents the chemo-sensitive group. PC, phosphatidylcholine. 
Table VI. Results of univariate logistic regression analysis of metabolites.

\begin{tabular}{|c|c|c|c|c|c|c|c|}
\hline \multirow[b]{2}{*}{ Clinicopathological variables } & \multirow[b]{2}{*}{$\mathrm{B}$} & \multirow[b]{2}{*}{ S.E. } & \multirow[b]{2}{*}{ Wald } & \multirow[b]{2}{*}{ P-value } & \multirow[b]{2}{*}{ Odds ratio } & \multicolumn{2}{|c|}{$95 \% \mathrm{CI}$} \\
\hline & & & & & & Lower & Upper \\
\hline Age, years & 0.394 & 0.676 & 0.339 & 0.560 & 1.482 & 0.394 & 5.579 \\
\hline Sex & -0.037 & 0.027 & 1.806 & 0.179 & 0.964 & 0.913 & 1.017 \\
\hline $\mathrm{T}$ classification & -0.118 & 0.608 & 0.038 & 0.846 & 0.889 & 0.27 & 2.926 \\
\hline Node classification & 0.044 & 1.445 & 0.001 & 0.975 & 1.045 & 0.062 & 17.765 \\
\hline Metastasis classification & -0.272 & 0.654 & 0.173 & 0.678 & 0.762 & 0.211 & 2.745 \\
\hline Differentiation & 0.118 & 0.608 & 0.038 & 0.846 & 1.125 & 0.342 & 3.703 \\
\hline Tumor size, $\mathrm{cm}$ & -0.224 & 0.702 & 0.102 & 0.749 & 0.799 & 0.202 & 3.163 \\
\hline Tumor location & 0.965 & 0.603 & 2.565 & 0.109 & 2.625 & 0.806 & 8.551 \\
\hline Vascular invasion & -1.068 & 1.211 & 0.778 & 0.378 & 0.344 & 0.032 & 3.688 \\
\hline Deoxyribose 1-phosphate & 0.000 & 0.000 & 13.363 & $<0.001^{\mathrm{c}}$ & 1.000 & 0.999 & 1.000 \\
\hline S-Lactoylglutathione & 0.003 & 0.001 & 11.195 & $0.001^{\mathrm{c}}$ & 1.003 & 1.001 & 1.004 \\
\hline LysoPC(16:0) & -0.002 & 0.002 & 12.639 & $0.002^{\mathrm{b}}$ & 0.999 & 0.999 & 1.000 \\
\hline O-Arachidonoyl Ethanolamine & -0.003 & 0.001 & 4.478 & $0.034^{\mathrm{a}}$ & 0.997 & 0.995 & 1.000 \\
\hline
\end{tabular}

${ }^{\mathrm{a}} \mathrm{P}<0.05,{ }^{\mathrm{b}} \mathrm{P}<0.01,{ }^{\mathrm{c}} \mathrm{P}<0.001 . \mathrm{PC}$, phosphatidylcholine; $\mathrm{B}$, regression coefficients; S.E, standard error; Wald, (B/S .E) ${ }^{2}$; OR, odds ratio; CI, confidence interval.

Table VII. Results of multivariate logistic regression analysis of metabolites.

\begin{tabular}{|c|c|c|c|c|c|c|c|}
\hline \multirow[b]{2}{*}{ Metabolite } & \multirow[b]{2}{*}{$\mathrm{B}$} & \multirow[b]{2}{*}{ S.E. } & \multirow[b]{2}{*}{ Wald } & \multirow[b]{2}{*}{ P-value } & \multirow[b]{2}{*}{ OR } & \multicolumn{2}{|c|}{$95 \% \mathrm{CI}$} \\
\hline & & & & & & Lower & Upper \\
\hline Deoxyribose 1-phosphate & 0.000 & 0.000 & 7.364 & $0.007^{\mathrm{b}}$ & 1.000 & 0.999 & 1.000 \\
\hline S-Lactoylglutathione & 0.002 & 0.001 & 5.057 & $0.025^{\mathrm{a}}$ & 1.002 & 1.000 & 1.003 \\
\hline
\end{tabular}

${ }^{\mathrm{a}} \mathrm{P}<0.05,{ }^{\mathrm{b}} \mathrm{P}<0.01$. B, regression coefficients; S.E, standard error; Wald, (B/S.E) ${ }^{2}$; OR, odds ratio; CI, confidence interval.

S-lactoylglutathione, lysoPC (16:0) and O-arachidonoyl ethanolamine. Use of these markers to differentiate between chemo-sensitive and chemo-resistant patients had a sensitivity and specificity of $82.5 \%$. Furthermore, multivariate regression analysis suggested that deoxyribose 1-phosphate and S-lactoylglutathione were independently and significantly associated with cancer chemosensitivity.

However, the present study did not determine the underlying biological mechanism for why alterations in the levels of these metabolites are associated with chemosensitivity, but there are a number of possible explanations. Deoxyribose 1-phosphate is an intermediate during pyrimidine metabolism (37) and functions as a substrate for thymidine phosphorylase (38). A previous study of gastric cancer reported that expression of mRNA for thymidine phosphorylase was higher in patients with lymph node metastasis compared with in patients without metastasis after chemotherapy treatment (39), suggesting that chemotherapy may affect thymidine phosphorylase-mediated metabolic pathways. Therefore, future studies should examine the association of deoxyribose 1-phosphate with thymidine phosphorylase in chemo-sensitive patients.

S-lactoylglutathione functions in the metabolism of pyruvate and can be hydrolyzed by hydroxyacyl glutathione hydrolase into D-lactic acid and glutathione, a substrate of glutathione S-transferase in the cytosol, microsomes and mitochondria (40). A single study have demonstrated that expression of glutathione S-transferase P1 is associated with the sensitivity of patients with gastric cancer to platinum-based therapies (41). The present study found that a lower serum level of S-lactoylglutathione was associated with chemosensitivity; however, it remains unclear whether this is due to the high expression of glutathione S-transferase P1. Future studies are required to examine these underlying mechanisms before these markers can be recommended for clinical diagnostic use.

The present study revealed that lysoPC was associated with chemotherapeutic sensitivity to gastric cancer. The overall level of PC is elevated in colorectal cancer (CRC) $(42,43)$. Kurabe et al (44) demonstrated that lysophosphatidylcholine acyltransferase 4 (LPCAT4) contributes to PC (16:0/16:1) accumulation in CRC via enhanced acylation of lysoPC. LPCAT4 is the factor responsible for the increase of PC (16:0/16:1) in CRC. The mechanism underlying the role of lysoPC in enhancing chemotherapeutic sensitivity in gastric cancer requires further investigation.

The present study revealed the differences in metabolites associated with chemosensitivity in patients who had 
unresectable gastric cancer. To predict the sensitivity to chemotherapy, serum samples were collected prior to chemotherapy. Future metabolomics studies associated with the prognosis of patients with unresectable gastric cancer receiving chemotherapy will be conducted.

The 47 patients in the present study included 13 patients with stage III gastric cancer, who were unable to accept stage I $\mathrm{R} 0$ resection due to local tumor surrounding important blood vessels. Stage III, peritoneal metastasis and liver metastasis are unresectable factors in patients with gastric cancer (45). Due to the limited number of samples in the present study, further analysis of different unresectable factors like liver, peritoneum metastases, lung and bone metastases was not conducted. In the future, the difference of metabolites between patients with hepatic and peritoneal metastatic gastric cancer should be studied. Future studies will include a larger sample size and will research the association between different unresectable factors and efficacy of chemotherapy for gastric cancer.

In summary, this metabolomic analysis of serum samples from patients with gastric cancer identified deoxyribose 1-phosphate, S-lactoylglutathione, lysoPC (16:0), and $\mathrm{O}$-arachidonoyl ethanolamine as metabolites that can differentiate chemo-sensitive and chemo-resistant patients. Additionally, the present study demonstrated that deoxyribose 1-phosphate and S-lactoylglutathione were significantly and independently associated with cancer chemosensitivity and, therefore, may serve as potential biomarkers.

\section{Acknowledgements}

Not applicable.

\section{Funding}

The present study was supported by the Foundation of Jilin Scientific and Technological Development Program (grant no. 20170623092TC-08).

\section{Availability of data and materials}

The datasets used and/or analyzed during the current study are available from the corresponding author on reasonable request.

\section{Authors' contributions}

JS, SZ and DW contributed equally in conceiving the hypothesis and overall study. design. YD collected the clinical data. WL, YD and LY analyzed and summarizing the final data. DW, SZ and JS contributed to the preparation of the manuscript. SZ and DW made great contributions in revising the paper for important intellectual content. All authors read and approved the final manuscript.

\section{Ethics approval and consent to participate}

The present study was approved by the Ethics Committee of First Hospital of Jilin University. Participants provided written informed consent.

\section{Patient consent for publication}

Not applicable.

\section{Competing interests}

The authors declare that they have no competing interests.

\section{References}

1. Chen WQ, Li H, Sun KX, Zheng RS, Zhang SW, Zeng HM, Zou XN, Gu XY and He J: Report of cancer incidence and mortality in China, 2014. Zhonghua Zhong Liu Za Zhi 40: 5-13, 2018 (In Chinese).

2. Li G, Chen X, Yu J and Liu H: Clinical research status of laparoscopic gastric cancer surgery in China, Japan and South Korea. Zhonghua Wei Chang Wai Ke Za Zhi 21: 126-131, 2018 (In Chinese).

3. Torre LA, Bray F, Siegel RL, Ferlay J, Lortet-Tieulent J and Jemal A: Global cancer statistics, 2012. CA Cancer J Clin 65: 87-108, 2015.

4. Zhang Q, Xu Z and Chen JF: Progress in the drug treatment of gastric cancer. Oncol Progress 12: 22-28, 2014

5. Sun J, Song Y, Wang Z, Chen X, Gao P, Xu Y, Zhou B and Xu H: Clinical significance of palliative gastrectomy on the survival of patients with incurable advanced gastric cancer: A systematic review and meta-analysis. BMC Cancer 13: 577, 2013.

6. Sougioultzis S, Syrios J, Xynos ID, Bovaretos N, Kosmas C, Sarantonis J, Dokou A, Tzivras D, Zografos G, Felekouras E, et al: Palliative gastrectomy and other factors affecting overall survival in stage IV gastric adenocarcinoma patients receiving chemotherapy: A retrospective analysis. Eur J Surg Oncol 37: 312-318, 2011.

7. Izuishi K and Mori H: Recent strategies for treating stage IV gastric cancer: Roles of palliative gastrectomy, chemotherapy, and radiotherapy. J Gastrointestin Liver Dis 25: 87-94, 2016.

8. Cunningham D, Allum WH, Stenning SP, Thompson JN Van de Velde CJ, Nicolson M, Scarffe JH, Lofts FJ, Falk SJ, Iveson TJ, et al: Perioperative chemotherapy versus surgery alone for resectable gastroesophageal cancer. N Engl J Med 355: 11-20, 2006.

9. Wilke H, Preusser P, Fink U, Gunzer U, Meyer HJ, Meyer J, Siewert JR, Achterrath W, Lenaz L, Knipp H, et al: Preoperative chemotherapy in locally advanced and nonresectable gastric cancer: A phase II study with etoposide, doxorubicin, and cisplatin. J Clin Oncol 7: 1318-1326, 1989.

10. Zuo CH, Ouyang YZ, Tang M, Tang W, Lin JG and Zhu HZ: Progress in comprehensive treatment of gastric cancer. Chin J Oper Proc Gen Surg 6: 319-324, 2012.

11. Ott K, Lordick F, Blank S and Buchler M: Gastric cancer: Surgery in 2011. Langenbecks Arch Surg 396: 743-758, 2011.

12. Furberg AS, Veierod MB, Wilsgaard T, Bernstein L and Thune I: Serum high-density lipoprotein cholesterol, metabolic profile, and breast cancer risk. J Natl Cancer Inst 96: 1152-1160, 2004.

13. Healy LA, Ryan AM, Carroll P, Ennis D, Crowley V, Boyle T, Kennedy MJ, Connolly E and Reynolds JV: Metabolic syndrome, central obesity and insulin resistance are associated with adverse pathological features in postmenopausal breast cancer. Clin Oncol (R Coll Radiol) 22: 281-288, 2010.

14. Mutoh M, Akasu T, Takahashi M, Niho N, Yoshida T, Sugimura T and Wakabayashi K: Possible involvement of hyperlipidemia in increasing risk of colorectal tumor development in human familial adenomatous polyposis. Jpn J Clin Oncol 36: 166-171, 2006.

15. Ychou M, Boige V, Pignon JP, Conroy T, Bouché O, Lebreton G, Ducourtieux M, Bedenne L, Fabre JM, Saint-Aubert B, et al: Perioperative chemotherapy compared with surgery alone for resectable gastroesophageal adenocarcinoma: An FNCLCC and FFCD multicenter phase III trial. J Clin Oncol 29: 1715-1721, 2011.

16. Furberg AS and Thune I: Metabolic abnormalities (hypertension, hyperglycemia and overweight), lifestyle (high energy intake and physical inactivity) and endometrial cancer risk in a Norwegian cohort. Int J Cancer 104: 669-676, 2003.

17. Backshall A, Sharma R, Clarke SJ and Keun HC: Pharmacometabonomic profiling as a predictor of toxicity in patients with inoperable colorectal cancer treated with capecitabine. Clin Cancer Res 17: 3019-3028, 2011. 
18. Stebbing J, Sharma A, North B, Athersuch TJ, Zebrowski A, Pchejetski D, Coombes RC, Nicholson JK and Keun HC: A metabolic phenotyping approach to understanding relationships between metabolic syndrome and breast tumour responses to chemotherapy. Ann Oncol 23: 860-866, 2012.

19. Wang D, Li W, Zou Q, Yin L, Du Y, Gu J and Suo J: Serum metabolomic profiling of human gastric cancer and its relationship with the prognosis. Oncotarget 8: 110000-110015, 2017.

20. Eisenhauer EA, Therasse P, Bogaerts J, Schwartz LH, Sargent D, Ford R, Dancey J, Arbuck S, Gwyther S, Mooney M, et al: New response evaluation criteria in solid tumours: Revised RECIST guideline (version 1.1). Eur J Cancer 45: 228-247, 2009.

21. Chen AP, Setser A, Anadkat MJ, Cotliar J, Olsen EA, Garden BC and Lacouture ME: Grading dermatologic adverse events of cancer treatments: The common terminology criteria for adverse events version 4.0. J Am Acad Dermatol 67: 1025-1039, 2012

22. Becker K, Mueller JD, Schulmacher C, Ott K, Fink U, Busch R, Böttcher K, Siewert JR and Höfler H: Histomorphology and grading of regression in gastric carcinoma treated with neoadjuvant chemotherapy. Cancer 98: 1521-1530, 2003.

23. Mandard AM,Dalibard F, Mandard JC, Marnay J, Henry-Amar M, Petiot JF, Roussel A, Jacob JH, Segol P, Samama G, et al: Pathologic assessment of tumor regression after preoperative chemoradiotherapy of esophageal carcinoma. Clinicopathologic correlations. Cancer 73: 2680-2686, 1994.

24. Mirza A, Naveed A, Hayes S, Formela L, Welch I, West CM and Pritchard S: Assessment of histopathological response in gastric and Gastro-oesophageal junction adenocarcinoma following neoadjuvant chemotherapy: Which scoring system to use? ISRN Pathol 2012: 1-8, 2012.

25. Ninomiya $Y$, Yanagisawa A, Kato $Y$, Kitagawa T, Ishihara S and Nakajima T: Histological indications of a favorable prognosis with far-advanced gastric carcinomas after preoperative chemotherapy. J Cancer Res Clin Oncol 125: 699-706, 1999.

26. Ferlay J, Shin HR, Bray F, Forman D, Mathers C and Parkin DM: Estimates of worldwide burden of cancer in 2008: GLOBOCAN 2008. Int J Cancer 127: 2893-2917, 2010.

27. Bray F, Ferlay J, Soerjomataram I, Siegel RL, Torre LA and Jemal A: Global cancer statistics 2018: GLOBOCAN estimates of incidence and mortality worldwide for 36 cancers in 185 countries. CA Cancer J Clin 68: 394-424, 2018.

28. Kinoshita J, Fushida S, Tsukada T, Oyama K, Okamoto K, Makino I, Nakamura K, Miyashita T, Tajima H, Takamura H, et al: Efficacy of conversion gastrectomy following docetaxel, cisplatin, and $\mathrm{S}-1$ therapy in potentially resectable stage IV gastric cancer. Eur J Surg Oncol 41: 1354-1360, 2015.

29. Sato Y, Ohnuma H, Nobuoka T, Hirakawa M, Sagawa T, Fujikawa K, Takahashi Y, Shinya M,Katsuki S, Takahashi M, et al: Conversion therapy for inoperable advanced gastric cancer patients by docetaxel, cisplatin, and S-1 (DCS) chemotherapy: A multi-institutional retrospective study. Gastric Cancer 20: 517-526, 2017.

30. Tsunematsu M, Takahashi N, Murakami K, Misawa T, Akiba T and Yanaga K: Successful conversion surgery for gastric cancer with multiple liver metastases treated after S-1 plus cisplatin combination chemotherapy: A case report. Surg Case Rep 3: 95 , 2017.

31. Lasithiotakis K, Antoniou SA, Antoniou GA, Kaklamanos I and Zoras O: Gastrectomy for stage IV gastric cancer. A systematic review and meta-analysis. Anticancer Res 34: 2079-2085, 2014.

32. Park SC and Chun HJ: Chemotherapy for advanced gastric cancer: Review and update of current practices. Gut Liver 7 : 385-393, 2013
33. Kawada K, Toda K and Sakai Y: Targeting metabolic reprogramming in KRAS-driven cancers. Int J Clin Oncol 22: 651-659, 2017.

34. Nitta H, Kelly BD, Allred C, Jewell S, Banks P, Dennis E and Grogan TM: The assessment of HER2 status in breast cancer: the past, the present, and the future. Pathol Int 66: 313-324, 2016

35. Matsuoka T and Yashiro M: Recent advances in the HER 2 targeted therapy of gastric cancer. World J Clin Cases 3: 42-51, 2015.

36. Xu J, Chen Y, Zhang R, Song Y, Cao J, Bi N, Wang J, He J, Bai J, Dong L, et al: Global and targeted metabolomics of esophageal squamous cell carcinoma discovers potential diagnostic and therapeutic biomarkers. Mol Cell Proteomics 12: 1306-1318, 2013.

37. Salway JG: Metabolism at a Glance. 3rd edition. Wiley-Blackwell, Oxford, p128, 2013.

38. Nishibeppu K, Komatsu S, Ichikawa D, Kosuga T, Okamoto K, Arita T, Konishi H, Morimura R, Murayama Y, Shiozaki A, et al: Long-term complete response of peritoneal recurrence from advanced gastric cancer using CapeOx therapy following radical gastrectomy. Gan To Kagaku Ryoho 44: 1173-1175, 2017 (In Japanese).

39. Li T, Liang M, Yuan J, Guo X, Feng D, Li T, Teng D, Peng Z, Wu X, Li Z, et al: Correlated analysis of 5 fluorouracil metabolic enzymes with tumor response after SOX regimen neoadjuvant chemotherapy in advanced gastric cancer. Zhonghua Yi Xue Za Zhi 94: 127-130, 2014 (In Chinese).

40. Gray LR, Tompkins SC and Taylor EB: Regulation of pyruvate metabolism and human disease. Cell Mol Life Sci 71: 2577-2604, 2014.

41. Shen X, Wang J, Yan X, Ren X, Wang F, Chen X and Xu Y: Predictive value of GSTP1 Ile105Val polymorphism in clinical outcomes of chemotherapy in gastric and colorectal cancers: A systematic review and meta-analysis. Cancer Chemother Pharmacol 77: 1285-1302, 2016.

42. Dobrzynska I, Szachowicz-Petelska B, Sulkowski S and Figaszewski Z: Changes in electric charge and phospholipids composition in human colorectal cancer cells. Mol Cell Biochem 276: 113-119, 2005.

43. Dueck DA, Chan M, Tran K, Wong JT, Jay FT, Littman C, Stimpson R and Choy PC: The modulation of choline phosphoglyceride metabolism in human colon cancer. Mol Cell Biochem 162: 97-103, 1996.

44. Kurabe N, Hayasaka T, Ogawa M, Masaki N, Ide Y, Waki M, NakamuraT,Kurachi K, Kahyo T, Shinmura K, etal: Accumulated phosphatidylcholine (16:0/16:1) in human colorectal cancer; possible involvement of LPCAT4. Cancer Sci 104: 1295-1302, 2013.

45. Yamaguchi K, Yoshida K, Tanahashi $\mathrm{T}$, Takahashi $\mathrm{T}$, Matsuhashi N, Tanaka Y, Tanabe K and Ohdan H: The long-term survival of stage IV gastric cancer patients with conversion therapy. Gastric Cancer 21: 315-323, 2018.

46. Ajani JA, D'Amico TA, Almhanna K, Bentrem DJ, Chao J, Das P, Denlinger CS, Fanta P, Farjah F, Fuchs CS, et al: Gastric cancer, version 3.2016, NCCN clinical practice guidelines in oncology. J Natl Compr Canc Netw 14: 1286-1312, 2016.

This work is licensed under a Creative Commons Attribution-NonCommercial-NoDerivatives 4.0 International (CC BY-NC-ND 4.0) License. 